Gynäkologe 2011 · 44:346-346

DOI 10.1007/s00129-010-2693-3

Online publiziert: 29. April 2011

(c) Springer-Verlag 2011

\author{
D. Berg ${ }^{1} \cdot \mathrm{K}$. Ulsenheimer ${ }^{2}$ \\ ${ }^{1}$ Amberg \\ ${ }^{2}$ München
}

\title{
Rechtliche Auflagen in Klinik und Praxis
}

Die berufsfachlichen Anforderungen an die Tätigkeit des Arztes in Klinik und Praxis werden ergänzt - und zum Teil übertroffen - durch vielfältige, immer wieder neue Auflagen des nahezu pausenlos tätigen Gesetzgebers und strenge Vorgaben einer von jährlich über 10.000 Klagen in Schwung gehaltenen, außerordentlich einflussreichen Haftungsrechtsprechung. Hier eine gewisse Übersicht in einigen Teilbereichen zu schaffen, ist Aufgabe dieses Themenheftes.

Viele sogenannte Behandlungsfehler gründen sich nicht auf eine ausschließlich ärztliche Fehlbehandlung, sondern auf Mängel der Rahmenbedingungen und vor allem der Organisation. In den Beiträgen von Herrn Berg und Frau Gaibler sowie Herrn Felber werden zum einen die administrativen Belastungen dargestellt, zum anderen Ansätze zur Problemlösung aufgezeigt. In seinem Beitrag beschäftigt sich Herr Selbmann mit der Sinnhaftigkeit der gesetzlichen Auflagen für Qualitätssicherung und Qualitätsmanagement im Sinne der Patientensicherheit. Die praktischen Auswirkungen stellen die Herren Staufer, Vater und Sohn, Arzt und Jurist, dar. Es wird gezeigt, dass bei allem Verständnis für rechtliche Forderungen die Umsetzung unter Beibehaltung von Standards und Patientenzuwendung auf ganz erhebliche Schwierigkeiten stößt. Herr Ulsenheimer beschreibt die rechtlichen Anforderungen an die persönliche Leistungserbringung im Krankenhaus und zeigt die Grenzen von Delegation bzw. Stellvertretung in diesem Bereich auf. In einem weiteren Beitrag bespricht er den heutigen Stand von Gesetzgebung und Rechtsprechung zur Patientenverfügung, Sterbehilfe und zum Behandlungsabbruch.
Was in diesem Themenheft aus Platzgründen fehlt, nämlich das Thema Aufklärung und Dokumentation, wird in einem anderen Heft publiziert werden. Das vorliegende Heft versucht, dem Arzt Hilfen zu geben, damit er sich im vermeintlichen Dschungel der rechtlichen Ansprüche zurechtfindet. Der Leser wird sich bewusst, dass fachärztliches Wissen nur der unabdingbare Grundstock der erforderlichen Kenntnisse ist. In der Realität der ärztlichen Tätigkeit sind die Kenntnisse der rechtlichen Anforderungen und die Fähigkeit zur organisatorischen Umsetzung genauso wichtig - zum Schutz der Patientin vor ihrem Arzt und des Arztes vor der Rechtsprechung.

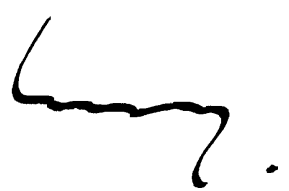

Prof. Dr. Dietrich Berg

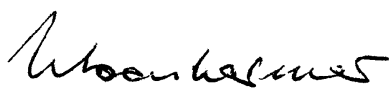

Prof. Dr. Klaus Ulsenheimer

\section{Korrespondenzadresse \\ Prof. Dr. D. Berg}

Schwaigerstr. 33, 92224 Amberg

dberg@asamnet.de 